\title{
Transcranial Magnetic Stimulation of the Medial Prefrontal Cortex Decreases Emotional Memory Schemas
}

\author{
Leonore Bovy ${ }^{1}$, Ruud M.W.J. Berkers ${ }^{1,2}$, Julia C.M. Pottkämper ${ }^{1,3}$, \\ Rathiga Varatheeswaran ${ }^{1,4}$, Guillén Fernández ${ }^{1}$, Indira Tendolkar ${ }^{1,5}$ and \\ Martin Dresler ${ }^{1}$
}

${ }^{1}$ Donders Institute for Brain, Cognition and Behaviour, Radboud University Medical Center, Nijmegen, The Netherlands, ${ }^{2}$ Max Planck Institute for Human Cognitive \& Brain Sciences, Leipzig, Germany, ${ }^{3}$ Clinical Neurophysiology, Institute for Technical Medicine, University of Twente, Enschede, The Netherlands, ${ }^{4}$ Leibniz Institute for Resilience Research, Mainz, Germany and ${ }^{5}$ Department of Psychiatry, Radboud University Medical Centre, Nijmegen, The Netherlands

*Address correspondence to Leonore Bovy, Kapittelweg 29, 6525 EN, Nijmegen, The Netherlands. Email: 1.bovy@donders.ru.nl, leonore.bovy@radboudumc.nl

\begin{abstract}
Mood-congruent memory bias is a critical characteristic of depression, but the underlying neural mechanism is largely unknown. Negative memory schemas might enhance encoding and consolidation of negative experiences, thereby contributing to the genesis and perpetuation of depressive pathology. To investigate this relationship, we aimed to perturb medial prefrontal cortex ( $\mathrm{MPFC}$ ) processing, using neuronavigated transcranial magnetic stimulation (TMS) targeting the mPFC. Forty healthy volunteers first underwent a negative mood induction to activate negative schema processing after which they received either active inhibitory $(N=20)$ or control $(N=20)$ stimulation to the mPFC. Then, all participants performed the encoding of an emotional false memory task. Recall and recognition performance was tested the following morning. Polysomnographic data were recorded continuously during the night before and after encoding. We observed a significantly lower false recognition of negative critical lures following mPFC inhibition, but no differences in veridical memory. These findings were supported by reaction time data, showing a relative slower response to negative compared with positive critical lures. The current findings support previous causal evidence for a role of the mPFC in schema memory processing and further suggest a role of the MPFC in memory bias.
\end{abstract}

Key words: bias, depression, memory, schema, TMS

\section{Introduction}

One of the major cognitive symptoms in depression is a moodcongruent memory bias for negative information (De Raedt and Koster 2010; Disner et al. 2011), meaning that depressed individuals are more likely to remember negative material than those without depression (Bradley et al. 1995). According to Beck's influential cognitive model of depression, memory biases during acquisition and processing of information play 
an important role in the onset and maintenance of depression (Beck 1967). These biases even persist after apparent recovery (Gilboa and Gotlib 1997). Indeed, negative memories formed throughout lifetime form the basis of many mental disorders, including post-traumatic stress disorder, anxiety disorder, addiction, and in its comorbidities (Vrijsen et al. 2018).

Despite decades of research in establishing the existence and role of negative memory biases, the neural mechanisms underlying memory bias are still elusive and have mainly been attributed to the role of amygdala (Ramel et al. 2007) and its modulation of the hippocampus (Hamilton and Gotlib 2008). However, the newly revived concept of schema as applied in the cognitive neuroscience of memory (Bartlett 1932) may shed new light on the neural mechanisms underlying memory bias in depression.

Schema-related memory processing refers to the integration of newly acquired congruent information into an existing superordinate and adaptable knowledge structure, which leads to superior encoding and consolidation efficiency (Mcclelland 2013; Gilboa and Marlatte 2017; Fernández and Morris 2018). In simpler terms, new information that fits well into an existing congruent network of information (i.e., a schema) is generally remembered better (Tse et al. 2007). This benefit in learning is dependent on the schema's activation and accessibility (Bransford and Johnson 1972). Whereas the standard model of memory consolidation assumes an initial critical role of the medial temporal lobe (MTL) and hippocampus and a subsequent gradual integration into neocortical representations, recent literature has identified the medial prefrontal cortex (mPFC) as a key player in the immediate integration of novel memories into preexisting knowledge structures (Kesteren, et al. 2010a, 2010b). Differential roles of the MPFC and the MTL in schema-dependent encoding were found, namely encoding-related activity in the mPFC increased linearly with increased congruency, whereas encoding-related MTL activity decreased (Kesteren et al. 2013). Additionally, it has been shown that patients who suffer from damage to the (ventro)mPFC show reduced schema representation (Ghosh et al. 2014; Warren et al. 2014). These findings indicate the necessity of the MPFC for schematic memory formation. In support of the causal role of the MPFC in schema memory formation, a recent neurostimulation study (Berkers et al. 2017) used transient magnetic stimulation (TMS) to perturb medial prefrontal processing before the performance of a Deese-Roedinger-McDermott (DRM) task (Roediger and Mcdermott 1995). In this false memory task, participants are presented with lists of semantically related words (e.g., tired, night, and pillow) that will establish a schema related to a specific nonpresented semantic associate, "the critical lure" (e.g., sleep). The beforementioned neurostimulation study (Berkers et al. 2017) showed that by applying a continuous theta burst stimulation (cTBS), the mPFC can be inhibited, leading to a reduction in false recall of critical lures, in line with studies on (v)mPFC lesioned patients (Warren et al. 2014). Note, however, that these findings are related to general knowledge structures. It is yet to be investigated how these findings transfer to emotional memory.

In depressed patients, previous studies have repeatedly shown an increased amygdala activity (Drevets et al. 1992; Siegle et al. 2007; Disner et al. 2011), a structure that is often thought to play a pivotal role in the consolidation of emotional memories (McGaugh 2004). In addition, particularly in chronic cases of depression, hippocampal function and structure shows progressive damage (MacQueen and Frodl 2011) and a reduction in size (Schmaal et al. 2016). Notably, increased MPFC activation is a further characteristic finding in depressed patients (Goldapple et al. 2004) and has been suggested as a biomarker for depression and its downregulation as a marker for therapeutic success (Farb et al. 2011; Lemogne et al. 2012). In support, a double dissociation of valence-specific response in an emotional go/no-go task was found in the rostral anterior cingulate cortex (ACC) extending to the anterior medial prefrontal cortex (Elliott et al. 2002). Specifically, this region was activated more strongly in response to sad words in depressed patients than to happy words, whereas this pattern was reversed in controls. An imbalance between MPFC and hippocampus, in combination with increased amygdala activity, may contribute to an increase in the recruitment of negatively toned memory schemas.

Behavioral evidence supporting this proposed mechanism comes from studies employing the DRM false memory task in depressed patients. Here, patients demonstrate a greater false memory for negative critical lures, corresponding to negative memory schema processing, in both free recall (Joormann et al. 2009) and recognition (Howe and Malone 2011). Similar results are found under negative mood induction conditions in healthy participants, where an induced negative mood leads to a preferential schema-like memory for negative critical lures (Ruci et al. 2009; Knott and Thorley 2014; Zhang et al. 2017).

Here, we propose schema memory processing as a candidate mechanism accounting for memory bias in depressed patients, who have particular strong negative schemas leading to an enhanced encoding and consolidation of negative experiences. Strong negative schemas may in fact account for the reduced tendency to process positive information even when in remission from depression (Arnold et al. 2012), thereby possibly accounting for the high relapse rate in depression.

Next to changes in memory function, patients also show alterations in sleep function (Palagini et al. 2013). And indeed, sleep is known to play an important role in memory consolidation (Stickgold 2005). The consolidation of both affective and schema-related memories has been associated with rapid eye moment (REM) sleep (Durrant et al. 2015; Genzel et al. 2015). Furthermore, neuroimaging studies have reported increased MPFC and amygdala activity during REM sleep (Maquet et al. 1996; Braun et al. 1997). REM sleep disinhibition has been considered a biological marker of depression, which is substantiated by decreased REM latency, increased REM duration, and increased REM density (Palagini et al. 2013). Consequently, increased REM sleep has been suggested to increase the consolidation of negative emotional memories in depression (Harrington et al. 2018). Importantly, the amount of REM sleep and prefrontal theta power during REM sleep has also been shown to predict both emotional (Nishida et al. 2009) and schematic memory consolidation (Durrant et al. 2015).

In sum, several lines of research point to an intimate relationship between negative memory bias, schema-related mPFC processing, and sleep. However, these three components have as of yet not been integrated. To provide further evidence for this relationship, we sought to perturb schema memory encoding using neuronavigated medial prefrontal stimulation, in line with a recent neurostimulation study (Berkers et al. 2017). In a sample of 40 healthy participants, we used a validated experimental approach for negative mood induction (Fitzgerald et al. 2011) to activate negative schema processing as an experimental model of depressed state. More specifically and in line with schema theories related to neutral memory (Bartlett 1932), mood induction should activate a transient negative schema, which should lead to a biased memory retrieval toward such negative 
schemas. We employed an adapted emotional version of the DRM false memory task to probe schema memory for both positive and negative critical lures. Lastly, we explored the relation of REM sleep to emotional and schema memory consolidation. In the registration of this study, we hypothesized first, that the active stimulation would diminish the number of total critical lures falsely remembered. We hypothesized, second, that the effect of the stimulation would specifically influence negative schemas due to their activation after the negative mood induction, resulting in a diminished negative memory bias in comparison to the control group. Third, we hypothesized that the amount of REM sleep and frontal theta power during REM sleep in the night between encoding and retrieval would be related to the number of critical lures that were falsely remembered.

\section{Methods and Materials}

\section{Participants}

Forty healthy native Dutch-speaking participants (26 females, 14 males, mean age 21.70 [SD \pm 3.33 ], ranging between 18 and 34 years) participated in return for course credit points or monetary compensation. All participants were right-handed, had normal or corrected-to-normal vision and no history of psychiatric or neurological illness as indicated through self-reporting. The study was conducted in accordance with the Declaration of Helsinki and approved by the local medical ethical committee (CMO Region Arnhem-Nijmegen, the Netherlands with Protocol ID: NL57736.091.16) and was registered at Netherlands Trial Register (NTR7080/Trial NL6893) on 18-03-2018 while data collection were ongoing, but before data analysis took place.

\section{Experimental Procedure}

The study consisted of three sessions on separate days. The first session was an intake session meant to collect baseline measurements, determine motor thresholds (MTs), and check for TMS tolerability. After providing written informed consent, participants were prescreened on any contraindication for TMS stimulation and prepared for the TMS intervention. This consisted of head registration for neuronavigation as well as resting and active MT determination. Then, a "practice" stimulation was performed to ensure participants were comfortable with the stimulation for the following day. This practice stimulation was identical in location, duration, and intensity to the actual stimulation they would receive the following day. Next, participants filled in baseline questionnaires (Becks Depression lnventory (BDI-I), State-Trait Anxiety Inventory (STAI-trait), NEO Five-Factor Inventory (NEO-FFI), Morningness-eveningness questionnaire (MEQ), Pittsburgh Sleep Quality Index (PSQI), St. Mary's Hospital sleep questionnaire (SMH) and Positive Affect Negative Affect Schedule (PANAS)). Finally, the portable sleep EEG system was attached for the baseline sleep recording. Intake sessions were always scheduled in the evening (after $6 \mathrm{pm}$ ), and participants were instructed to not partake in any rigorous activity for the rest of the evening after EEG application.

The following day, the first experimental session took place. First, participants were prepared for the TMS session, which included head registration for neuronavigation. Then, participants filled in questionnaires (SMH and PANAS). Next, negative mood was induced through watching several film clips. After the movie, a second PANAS was administered to probe the effectiveness of the mood induction. Immediately, thereafter, the participants received either cTBS $(N=20)$ or rTMS $(5 \mathrm{~Hz}$;
$\mathrm{N}=20$ ) stimulation for $40 \mathrm{~s}$, targeting the mPFC. In a neighboring behavioral lab, they performed the emotional DRM encoding task. Participants were instructed to remember as many words as possible and were reminded they would be tested the following day, similar to the original DRM instructions. Finally, a third PANAS was filled in, and the portable sleep EEG was again attached. The following day, during the second experimental session, participants freely recalled as many encoded words as possible, followed by a recognition task. After completing all tasks, subjects responded to debriefing questions on the purpose of the study. See Figure 1 for a schematic overview. The gray triangles represent the administration of a PANA questionnaire.

\section{False Memory Task}

An adapted version of the well-established Deese-RoedigerMcDermott (DRM) false memory task was used (Roediger and Mcdermott 1995). To probe the activation of emotional schemas and measure emotional memory bias, we constructed new emotional semantically associated lists using the Dutch version of the Affective Norms for English Words list (Arnold et al. 2011) in an initial pilot study. See Supplementary Materials for further details. Each list contained 10 words corresponding to one critical lure. Each word was presented twice (first in the order of strongest to weakest association with the critical lure, then immediately thereafter in semirandom order) on a computer screen for $600 \mathrm{~ms}$ with a $125 \mathrm{~ms}$ interstimulus interval. Twenty lists of 10 words each were presented during encoding, corresponding to 10 positive and 10 negative lists. In order to maximize the effect of interest (a bias for negative in contrast to positive stimuli) and due to the constraints of a time-limited window of stimulation effects, a neutral condition was omitted. During the free recall, participants were asked to provide confidence ratings (1-5) for each reported word, to maximally ensure they would write down as many words as possible. The participants were not given any time limit and on average took $26.14( \pm \mathrm{SD}=9.52) \mathrm{min}$. During the recognition task, three old, three new, and one lure items per list were semirandomly presented on the screen. Here, the participant had to indicate if the word was new or old and immediately provide a confidence rating (sure, somewhat sure, and unsure), within $3 \mathrm{~s}$. To disentangle the effects of specific sleep parameters on the consolidation of false memories, encoding and retrieval sessions took place on consecutive days with a full night of EEGrecorded sleep in between. This design is in line with previous studies probing the effect of sleep on false memory processing (e.g., Diekelmann et al. 2008).

\section{TMS Procedure}

The active cTBS intervention and procedure was an exact copy of the study by Berkers et al. (2017). The active cTBS stimulation is thought to work inhibitory (Huang et al. 2005), whereas the $5 \mathrm{~Hz}$ rTMS stimulation was chosen as our control stimulation based on previous literature (Volman et al. 2011) showing no behavioral difference with cTBS-vertex control stimulation in a similar set-up as our study. The cTBS stimulation protocol consisted of a total of 600 pulses administered across $40 \mathrm{~s}$, which consisted of bursts of three pulses at $50 \mathrm{~Hz}$, and each burst was repeated at a frequency of $5 \mathrm{~Hz}$. The control rTMS stimulation protocol consisted of a total of 200 pulses, administered across $40 \mathrm{~s}$, at a frequency of $5 \mathrm{~Hz}$. Stimulation intensity was set at $80 \%$ of the active motor threshold from the tibialis anterior. 

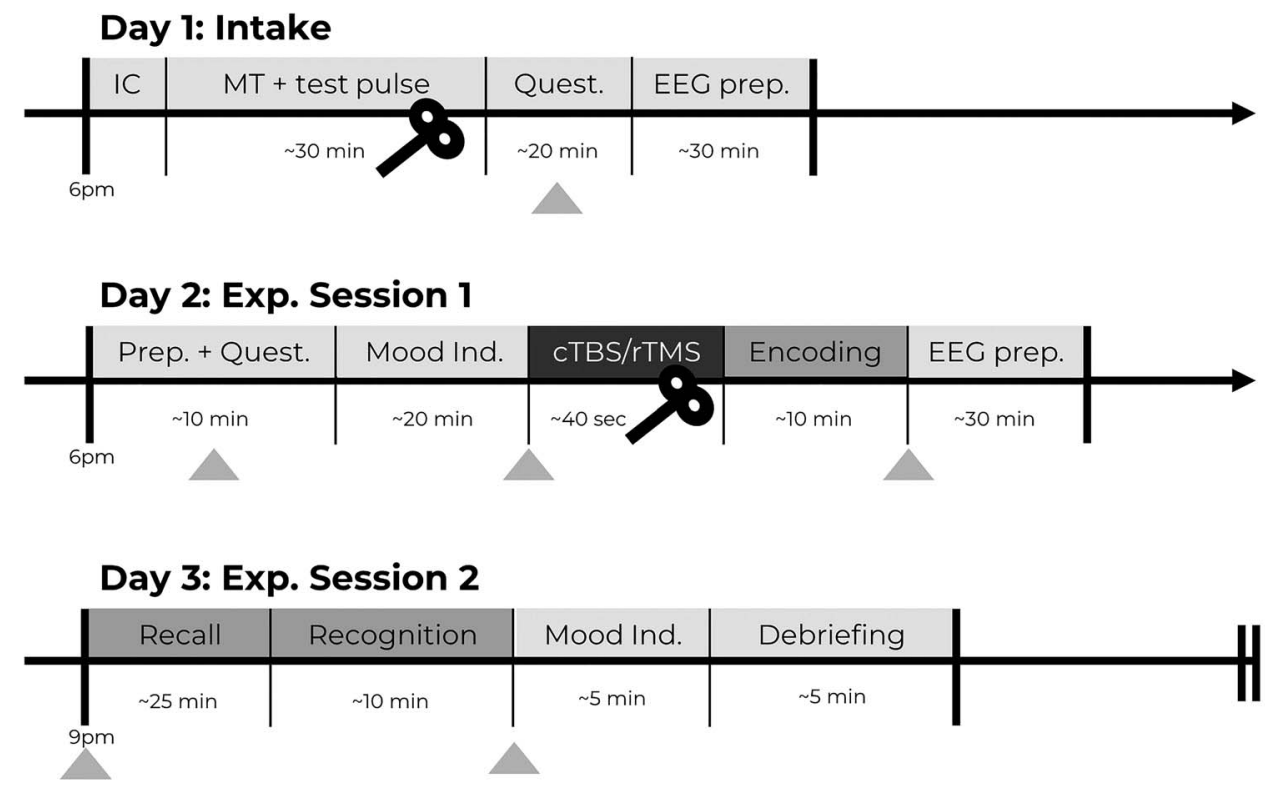

Figure 1. Experimental procedure. Overview of procedures during the three testing days. The gray triangles represent the administration of a PANAS questionnaire.

See Supplementary Materials for further details on the TMS procedure.

\section{Mood Induction}

Sad mood was induced in all participants by presenting segments of an emotional movie (Westermann et al. 1996), in order to activate negative schema memory processing, and thereby imitating a negative memory bias as seen in depressed patients (Joormann et al. 2009). Two consecutive segments of the movie "Sophie's Choice" were presented for $20 \mathrm{~min}$ [adapted from Fitzgerald et al. (2011)]. See Supplementary Materials for participant instructions. At the end of the final experimental session, a short segment from the movie "Happy Feet" was presented to induce a positive mood and to uplift any possibly lingering negative moods (Fitzgerald et al. 2011).

\section{Sleep Recordings}

Overnight polysomnographic sleep recordings were made for two consecutive nights at the participants' home, using a 15channel Somnoscreen ${ }^{\mathrm{TM}}$ plus (SOMNOmedics $\mathrm{GmbH}$, Randersacker, Germany) portable device. Electroencephalography (EEG) measurements were recorded with gold cup electrodes at six EEG locations (F3, F4, C3, C4, O1, and O2) and were attached according to the American Academy of Sleep Medicine rules (Berry et al. 2015). See Supplementary Materials for further details on the sleep recordings.

\section{Analysis}

All analyses were conducted in the $\mathrm{R}$ programming language (version 3.5.1; R Core Team 2018). The differences between the two experimental groups were analyzed through two-tailed Welch's t-tests for unequal variances, unless otherwise stated. For the false memory task, the hit, false alarm, and criticallure rates were calculated. A discrimination ability index (dprime) was calculated as follows: $d^{\prime}=z(H)-z(F A)$. A higher value indicates that a subject had greater ability to discriminate between studied and unstudied items. In addition, a d-prime value on the sensitivity to falsely recognizing critical lures was calculated as follows: $d{ }^{\prime}$ criticallures $=z\left(F A_{\text {criticallures }}\right)-z(F A)$. Here, higher values suggest a higher propensity to falsely recognize lures. In addition, we analyzed the ratio between negative and positive critical lures as a measure of emotional biased responses. Tukey's ladder of powers was applied when data were found to be non-normal.

\section{Results}

\section{Mood Induction}

The results of the PANAS questionnaires confirmed the effectiveness of the negative mood induction on the participants' mood. See Supplementary Materials for further details.

\section{Recall Results}

Subjects correctly recalled on average 29.60 (SD \pm 11.76 ) words out of all 200 studied items. No ratio difference between positive and negative items was observed for old items, $t(39)=-1.50$, $P=0.14,95 \% C I=[0.58,1.06], d=0.24$.

Subjects falsely recalled on average $6.22(\mathrm{SD} \pm 3.19)$ out of 20 total lures. No ratio difference between positive and negative critical lures was found, $t(36.97)=-1.37, P=0.18,95 \%$ $\mathrm{CI}=[-0.42,0.08], d=0.35$. The two groups did neither differ in the number of total old items remembered, $t(35.32)=0.99$, $P=0.33,95 \% C I=[-3.85,11.25], d=0.31$, nor in the ratio of old items, $t(29.49)=0.25, P=0.81,95 \% C I=[-0.06,0.08], d=0.13$, cTBS: $M=5.80, S D \pm 2.33$, rTMS: $M=6.65, S D \pm 3.88$.

The groups did neither differ in the total number of critical lures falsely remembered, $t(31.11)=0.84, P=0.41,95 \% \mathrm{CI}=[-1.22$, 2.92], $d=0.27$, nor in the ratio of negative and positive critical lures, $t(32.91)=-1.08, P=0.29,95 \% C I=[-0.76,0.24], d=0.35$.

\section{Recognition Results}

Participants recognized old words better than chance (mean dprime old item $=0.60$, versus chance level d-prime $=0, t(39)=7.57$, $P<0.001,95 \% C I=[0.44,0.76], d=1.20)$. In addition, endorsement 


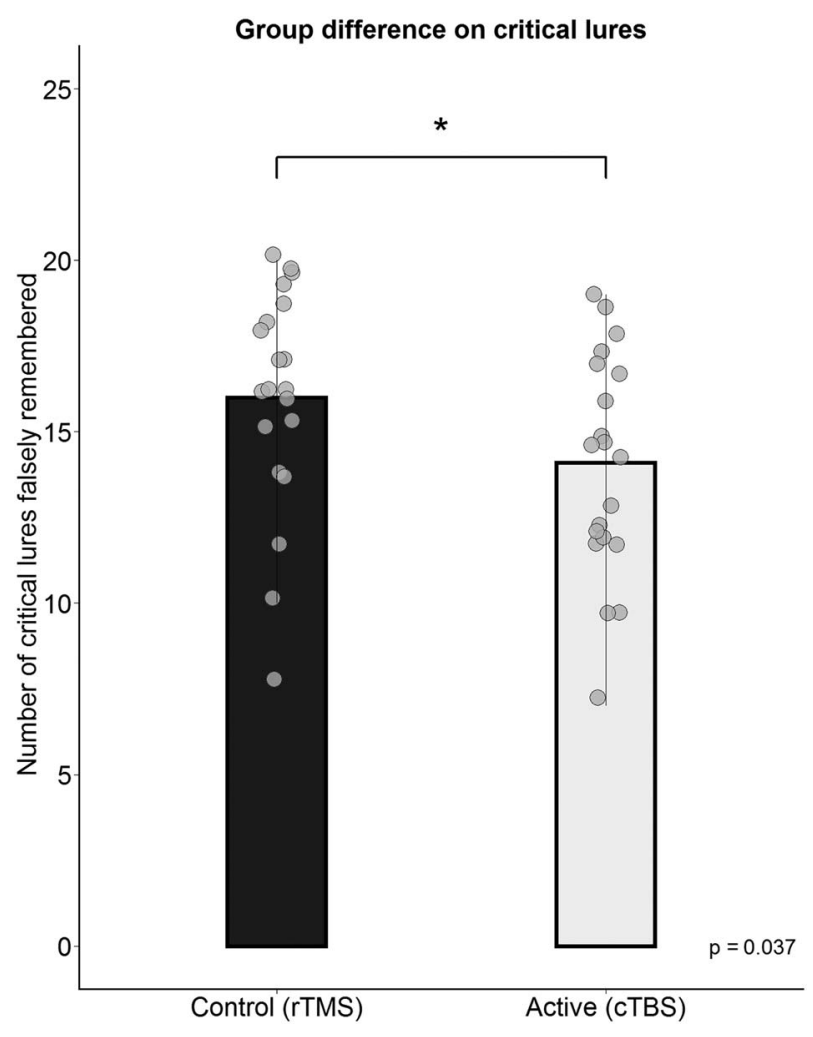

Figure 2. Group differences on the recognition of critical lures. A significant difference between the control and active stimulation group was found, where the active group falsely remembered on average less critical lures than the control group *,$p<.05$.

of critical lures as old studied items was also higher than chance (mean d-prime critical lures $=0.93$, versus chance level $d$-prime $=0, t(39)=7.82, P<0.001), 95 \% C I=[0.69,1.17], d=1.24$. No ratio difference in hit rates between positive and negative items was observed for old items, $t(39)=-0.76, P=0.45,95 \%$ $\mathrm{CI}=[0.88,1.05], d=0.12$, nor for critical lures, $t(39)=1.70, P=0.10)$, $95 \% \mathrm{CI}=[0.99,1.06], d=0.03$.

The d-prime scores did not differ between the two groups, $t(38.00)=0.51, P=0.61,95 \% C I=[-0.24,0.40], d=0.16$. Furthermore, the groups did not differ in ratio between hit rates between positive and negative items for old items, (rTMS: $M=1.00$, cTBS: $M=0.93$ ), after Tukey's ladder of powers was applied due to nonnormality, $\mathrm{t}(37.26)=0.88, P=0.38,95 \% \mathrm{CI}=[-0.02,0.06], d=0.45$. In accordance to our main hypotheses, the participants in the active stimulation (cTBS) group falsely remembered on average less lures, $(M=14.10, S D=3.26)$ than those in the control (rTMS) group, $(M=16, S D=3.26)$. This difference was statistically significant on a one-tailed $t$-test, $t(38.00)=1.84, P=0.04,95 \% \mathrm{CI}=[0.16$, Inf], $d=0.58$, see Figure 2. The $d$-prime scores for critical lures, however, did not reach statistical significance with a one-tailed t-test, $\mathrm{t}(34.29)=1.57, P=0.06,95 \% \mathrm{CI}=[-0.03, \operatorname{Inf}], d=0.50$.

More crucially (after removal of two outliers of two standard deviations from the mean in the CTBS group), the groups significantly differed in the ratio between the false alarm rates of positive and negative critical lures, $t(34.20)=2.27, P=0.03,95 \%$ $\mathrm{CI}=[0.02,0.35], d=0.03$.

The cTBS group showed a more positive tendency or bias (see Fig. $3 \mathrm{~A}$ ) than the rTMS group (rTMS: $M=1.01$, cTBS: $M=0.82$ ). These results were supported by reaction time (RT) data, showing a similar group difference in the ratio of the valenced response times (positive/negative lures $\mathrm{RT}$ ), $\mathrm{t}(33.48)=2.87$, $P=0.01,95 \% C I=[0.06,0.36], d=0.43$, see Figure $3 B$.

To explore the direction of the observed group difference in ratio scores, group differences between the false recognition of negative critical lures were tested in a posthoc t-test, revealing a decrease of false recognition of only negative critical lures in the active cTBS condition in comparison to the control condition, $t(37.87)=2.41, P=0.02,95 \% C I=[0.23,2.67], d=0.76$, see Figure 4.

\section{Sleep Data}

Frontal theta power during the night between encoding and retrieval was neither correlated with the amount of critical lures recognized, $b<0.01, \mathrm{t}(38)=-0.10, P=.919, \eta^{2}<0.01$, nor with the ratio between positive and negative critical lures recognized, $b=-0.01, \mathrm{t}(38)=-1.38, P=.176, \eta^{2}=0.05$. See Supplementary Materials for more sleep data results.

\section{Discussion}

The present study was designed to investigate whether (emotional) schema memory could be reduced by transiently perturbing the medial prefrontal cortex (mPFC) using theta burst transcranial magnetic stimulation (TMS). The results of the current study seem to support the involvement of the mPFC in schema memory processing, as inhibition of the MPFC with continuous theta burst stimulation (CTBS) decreased false recognition in the active experimental group compared with the control group. These findings are consistent with the results of a previous TMS study (Berkers et al. 2017) and with previous lesion studies (Warren et al. 2014). More importantly, after a negative mood induction, a group difference emerged in the ratio between positive and negative critical lures that were falsely recognized. Here, the active stimulation specifically decreased false recognition of negative lures. In support, the experimental groups also differed in the ratio of reaction times between negative and positive critical lure recognition, demonstrating that not only memory but also the speed of retrieval was affected by the stimulation.

The current findings show initial support to an association of mPFC-related emotional memory schemas and mood congruent memory bias. Indeed, other false memory studies using mood induction paradigms (Zhang et al. 2017) have found moodcongruent effect after a negative mood induction in healthy participants. Previous meta-analytic studies have shown that healthy nondepressed individuals show a positive memory bias (Matt et al. 1992; Gaddy and Ingram 2014), but a negative mood induction can induce a more negative memory bias (Ruci et al. 2009). We propose that in our sample, the negative mood induction activated negative schemas and induced a memory bias that is mood-congruent and thus more negative. Ultimately, this resulted in a noncommittal bias as we observed in the control stimulation group. In turn, we propose that the active inhibitory stimulation of the mPFC may have specifically abolished the effects of the negative mood induction by targeting the activated negative schemas, subsequently leading to a relatively more positive bias than the control group. On a neural level, emotional stimuli are relayed via the thalamus to the amygdala, where subsequently the amygdala has been proposed as a mediator for mood congruent memory processing (Ramel et al. 2007) and a modulator of hippocampal activity during emotional memory formation (Dolcos et al. 2005). Amygdalic activation is generally 

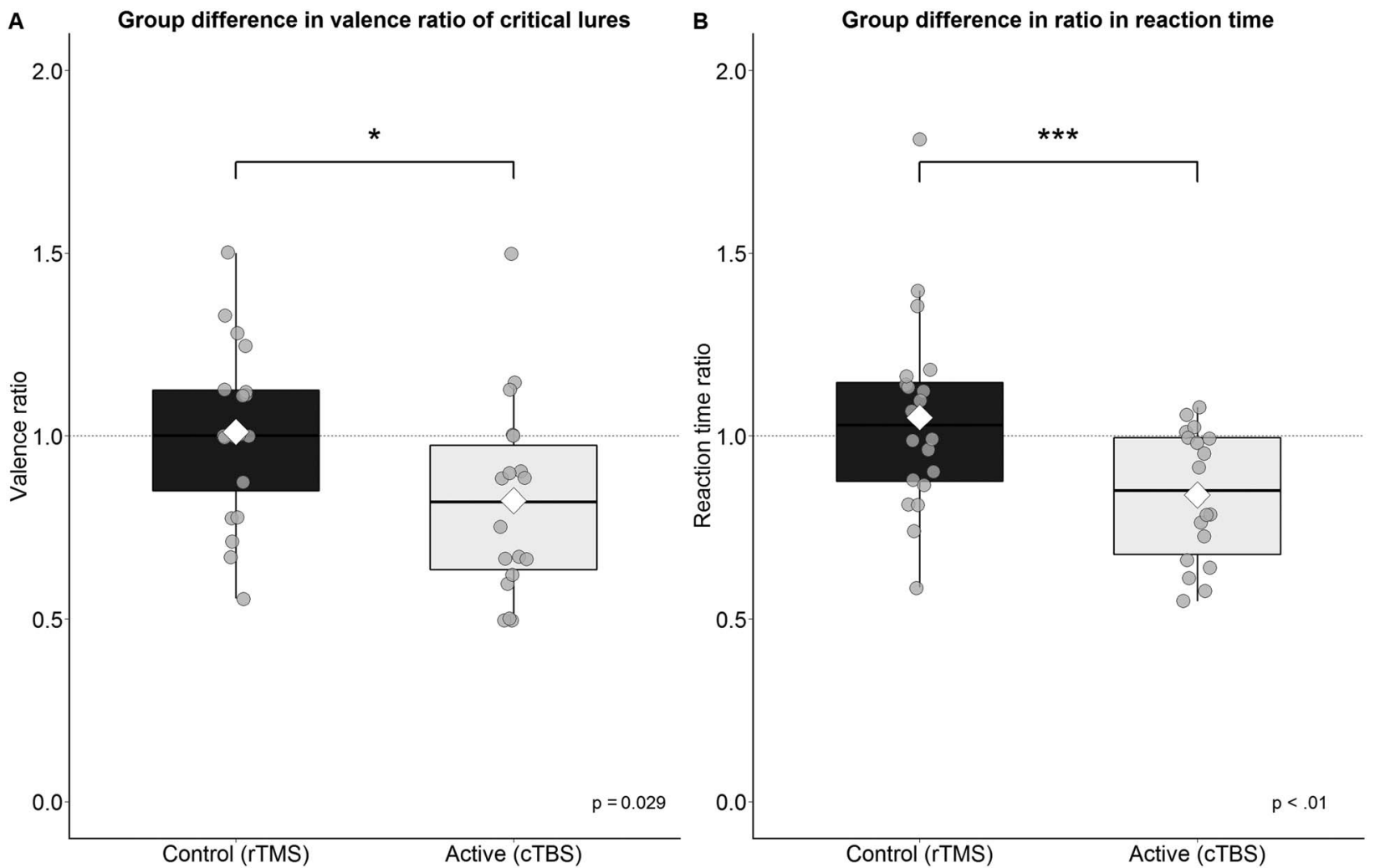

Figure 3. Group differences in the valence ratio and reaction time of the recognition of critical lures. (A) The active group on average falsely remembered less negative critical lures relative to positive in comparison to the control group. (B) The active group was on average faster in responding to positive items than negative items. The white diamond shape represents the mean ${ }^{*}, p<.05,{ }^{* * *}, p<.01$.

higher in patients in comparison to controls and receives less inhibition from the higher-order top-down dorsolateral PFC (Disner et al. 2011). Together with increased mPFC activity, increased amygdala activity and MPFC-amygdala connectivity might augment the reactivation of emotionally negative schemas. Functional MRI studies could specifically delineate the involvement of the amygdala in connectivity to the MPFC during the encoding of emotional memory schemas.

Schemas have been considered in the cognitive theory of depression (Beck 1967). Whereas in the neuroscience literature, schema is defined as a framework of knowledge that enables efficient memory storage (Gilboa and Marlatte 2017), Beck's definition of cognitive schemas includes mental representations of experiences, containing attitudes about oneself, which influence how information is being processed. These cognitive schemas in turn lead to specific memory biases in depression. We propose that these two notions are not mutually exclusive and show conceptual and neurobiological overlap. Indeed, we extend the current neurobiological model of depression (Disner et al. 2011) by suggesting that increased amygdala, hippocampus and MPFC reactivity in response to an aversive stimulus not only leads to increased ruminative thought or reinforcement of self-referential schemas but also increased negative memory schema processing and biased memory for negative stimuli.

Previous studies reported group differences in recall (Warren et al. 2014; Berkers et al. 2017), whereas we report differences in recognition. This discrepancy could be attributed to the fact that the standard DRM (false memory) task includes an immediate recall after each separate list and a recognition session immediately thereafter. Our task included $\mathrm{a}>12$ - $\mathrm{h}$ delay between encoding and both recall and recognition. Therefore, we could dissociate between the effects of the stimulation on encoding and retrieval but also increased task difficulty. A longer delay between encoding and retrieval has been shown to decrease false memory (Thapar and McDermott 2001). This delay could have removed any ceiling effects of the recognition results as was found in the previous studies which might have concealed group differences and could have made recall more difficult. In addition, the current study focused on the influence of MPFC stimulation before the encoding of a false memory task. Future studies should explore if similar effects can be found when stimulating before/during the retrieval phase in a similar design (Kensinger and Ford 2019). Indeed, such studies would be clinically very relevant given the greater manipulative control over retrieval of, for example, traumas or negative ruminations than over encoding.

There are, however, some caveats. First, our design did not include a neutral or positive mood condition. Therefore, no specific directional effect of the mood induction can be ascertained. Second, the current design included a control stimulation protocol of $5 \mathrm{~Hz}$ applied to the same mPFC location as the active stimulation to ensure a similar stimulation sensation in both our experimental groups. Given that one of our main hypotheses regarded responses to emotional stimuli, we aimed to exclude any group differences in mood due to potential differences in pain or discomfort. A control stimulation of $5 \mathrm{~Hz}$ was chosen based on a previous study by Volman et al. (2011), who found no behavioral differences between a $5 \mathrm{~Hz}$-anterior PFC or CTBS-vertex control stimulation in a similar set-up as our study. However, we cannot exclude the possibility that our control stimulation did excite the cortex, thus possibly driving the group 


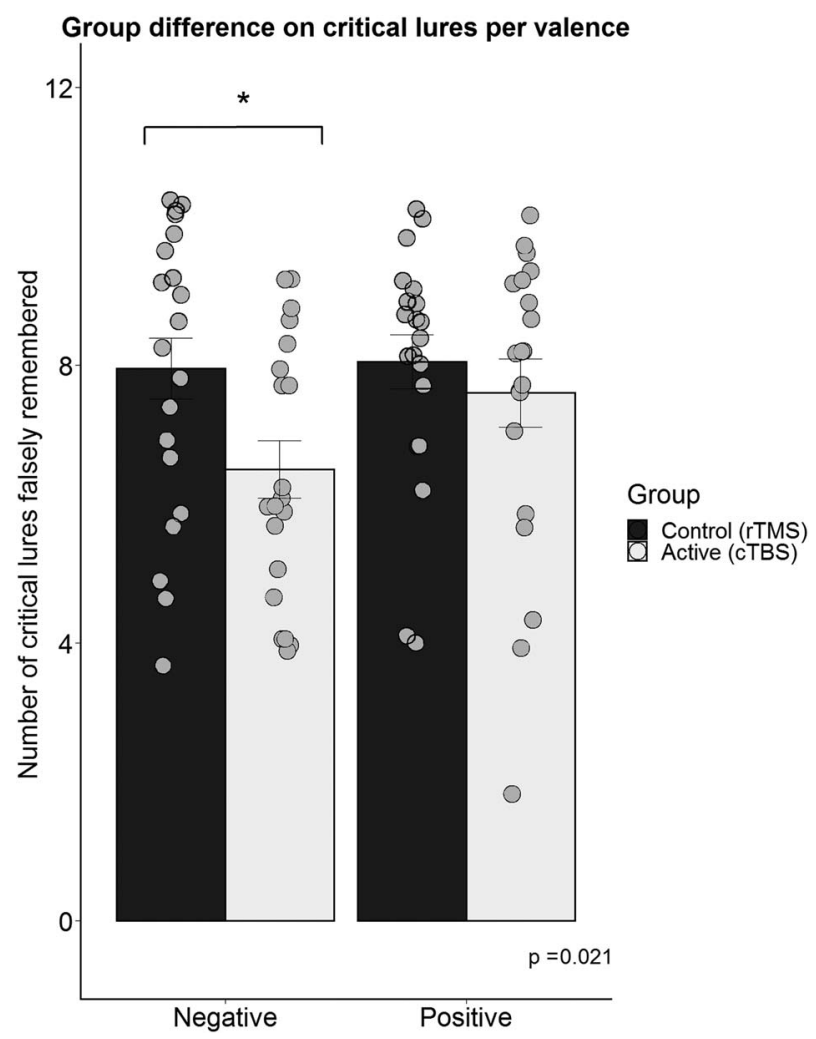

Figure 4. Group differences on the recognition of critical lures per valence. A group difference was found on the negative critical lures ${ }^{*}, p<.05$.

difference effect instead of the cTBS inhibition. This possibility is, however, not supported by the current literature on the involvement of the mPFC and memory processing. In addition, the application of the control stimulation to the same cortical location as the active stimulation limits the interpretation of the specificity of the effect. Future endeavors should include additional control stimulation sites to delineate the specificity of the mPFC as target. Third, the current stimulation protocol was optimized to stimulate deeper brain regions, targeting the mPFC. This, however, leads to additional stimulation of the areas between the stimulation coil and the target region. Berkers et al. (2017) discuss the impact of this factor and the uncertainty on the effective depth of the stimulation and will thus not be further discussed here. Lastly, to discern the directionality of the observed emotional biases compared with neutral conditions, future studies should additionally include neutral lists. In our design, no neutral lists were used due to time constraints of the encoding session, as the effect of the mood induction and brain stimulation would taper off with increased passing of time (Huang et al. 2005). Therefore, we decreased the number of lists of the encoding session by omitting the neutral condition and focused on the contrast between positive and negative stimuli to measure potential memory biases.

In summary, mPFC inhibition after negative schema activation appears to be able to specifically decrease negative schema memories. The ability to specifically target and diminish negative memories may be a valuable instrument for therapeutic purposes. Given these new findings, it would be fruitful to further explore the possibilities of manipulating emotional schemas.

\section{Supplementary Material}

Supplementary material is available at Cerebral Cortex online.

\section{Funding}

Intramural funding was obtained from the Donders Center for Medical Neuroscience.

\section{Notes}

We would like to thank all the study participants, as well as all the students and interns who helped during data acquisition for their support. Conflict of Interest: None declared.

\section{References}

Arnold JF, Fitzgerald DA, Fernández G, Rijpkema M, Rinck M, Eling PA, Becker ES, Speckens A, Tendolkar I. 2011. Rose or black-coloured glasses?: Altered neural processing of positive events during memory formation is a trait marker of depression. Journal of Affective Disorders. 131:214-223.

Arnold JF, Zwiers MP, Fitzgerald DA, van Eijndhoven P, Becker ES, Rinck M, Fernández G, Speckens AEM, Tendolkar I. 2012. Fronto-limbic microstructure and structural connectivity in remission from major depression. Psychiatry Research: Neuroimaging. 204:40-48.

Bartlett F. 1932. Remembering: an experimental and social study. Cambridge: Cambridge University.

Beck AT. 1967. Depression: clinical, experimental, and theoretical aspects. New York: Harper \& Row.

Berkers RMWJ, van der Linden M, de Almeida RF, Müller NCJ, Bovy L, Dresler M, Morris RGM, Fernández G. 2017. Transient medial prefrontal perturbation reduces false memory formation. Cortex. 88:42-52.

Berry RB, Gamaldo CE, Harding SM, Brooks R, Lloyd RM, Vaughn BV, Marcus CL. 2015. AASM scoring manual version 2.2 updates: new chapters for scoring infant sleep staging and home sleep Apnea testing. Journal of Clinical Sleep Medicine. 11:1253-1254.

Bradley BP, Mogg K, Williams R. 1995. Implicit and explicit memory for emotion-congruent information in clinical depression and anxiety. Behaviour Research and Therapy. 33:755-770.

Bransford JD, Johnson MK. 1972. Contextual prerequisites for understanding: some investigations of comprehension and recall. Journal of Verbal Learning and Verbal Behavior. 11:717-726.

Braun AR, Balkin TJ, Wesenten NJ, Carson RE, Varga M, Baldwin P, Selbie S, Belenky G, Herscovitch P. 1997. Regional cerebral blood flow throughout the sleep-wake cycle. An H2(15)O PET study. Brain. 120:1173-1197.

De Raedt R, Koster EHW. 2010. Understanding vulnerability for depression from a cognitive neuroscience perspective: a reappraisal of attentional factors and a new conceptual framework. Cognitive, Affective, \& Behavioral Neuroscience. 10:50-70.

Diekelmann S, Landolt H-P, Lahl O, Born J, Wagner U. 2008. Sleep loss produces false memories. PLOS One. 3:e3512.

Disner SG, Beevers CG, Haigh EAP, Beck AT. 2011. Neural mechanisms of the cognitive model of depression. Nature Reviews Neuroscience. 12:467-477.

Dolcos F, LaBar KS, Cabeza R. 2005. Remembering one year later: role of the amygdala and the medial temporal lobe memory 
system in retrieving emotional memories. Proceedings of the National Academy of Sciences. 102:2626-2631.

Drevets WC, Videen TO, Price JL, Preskorn SH, Carmichael ST, Raichle ME. 1992. A functional anatomical study of unipolar depression. Journal of Neuroscience. 12:3628-3641.

Durrant SJ, Cairney SA, McDermott C, Lewis PA. 2015. Schemaconformant memories are preferentially consolidated during REM sleep. Neurobiology of Learning and Memory, REM Sleep and Memory. 122:41-50.

Elliott R, Rubinsztein JS, Sahakian BJ, Dolan RJ. 2002. The neural basis of mood-congruent processing biases in depression. Archives of General Psychiatry. 59:597-604.

Farb NAS, Anderson AK, Bloch RT, Segal ZV. 2011. Moodlinked responses in medial prefrontal cortex predict relapse in patients with recurrent unipolar depression. Biological Psychiatry, Neural Connectivity, Ruminations, and Suicide. 70: 366-372.

Fernández G, Morris RGM. 2018. Memory, novelty and prior knowledge. Trends in Neurosciences, Special Issue: Time in the Brain. 41:654-659.

Fitzgerald DA, Arnold JF, Becker ES, Speckens AEM, Rinck M, Rijpkema M, Fernández G, Tendolkar I. 2011. How mood challenges emotional memory formation: an fMRI investigation. NeuroImage. 56:1783-1790.

Gaddy MA, Ingram RE. 2014. A meta-analytic review of moodcongruent implicit memory in depressed mood. Clinical Psychology Review. 34:402-416.

Genzel L, Spoormaker VI, Konrad BN, Dresler M. 2015. The role of rapid eye movement sleep for amygdala-related memory processing. Neurobiology of Learning and Memory, REM Sleep and Memory. 122:110-121.

Ghosh VE, Moscovitch M, Colella BM, Gilboa A. 2014. Schema representation in patients with ventromedial PFC lesions. Journal of Neuroscience. 34:12057-12070.

Gilboa A, Marlatte H. 2017. Neurobiology of schemas and schema-mediated memory. Trends in Cognitive Sciences. 21:618-631.

Gilboa E, Gotlib IH. 1997. Cognitive biases and affect persistence in previously dysphoric and never-dysphoric individuals. Cognition and Emotion. 11:517-538.

Goldapple K, Segal Z, Garson C, Lau M, Bieling P, Kennedy S, Mayberg H. 2004. Modulation of cortical-limbic pathways in major depression: treatment-specific effects of cognitive behavior therapy. Archives of General Psychiatry. 61:34-41.

Hamilton JP, Gotlib IH. 2008. Neural substrates of increased memory sensitivity for negative stimuli in major depression. Biological Psychiatry, Towards Personalized Treatment for Depression. 63:1155-1162.

Harrington MO, Johnson JM, Croom HE, Pennington K, Durrant SJ. 2018. The influence of REM sleep and SWS on emotional memory consolidation in participants reporting depressive symptoms. Cortex. 99:281-295.

Howe ML, Malone C. 2011. Mood-congruent true and false memory: effects of depression. Memory. 19:192-201.

Huang Y-Z, Edwards MJ, Rounis E, Bhatia KP, Rothwell JC. 2005. Theta burst stimulation of the human motor cortex. Neuron. 45:201-206.

Joormann J, Teachman BA, Gotlib IH. 2009. Sadder and less accurate? False memory for negative material in depression. Journal of Abnormal Psychology. 118:412-417.

Kensinger EA, Ford JH . 2019. Retrieval of emotional events from memory. Annual review of psychology. 71. van Kesteren MTR, Beul SF, Takashima A, Henson RN, Ruiter DJ, Fernández G. 2013. Differential roles for medial prefrontal and medial temporal cortices in schema-dependent encoding: from congruent to incongruent. Neuropsychologia, Special Issue on Functional Neuroimaging of Episodic Memory. 51:2352-2359.

van Kesteren MTR, Fernández G, Norris DG, Hermans EJ. 2010a. Persistent schema-dependent hippocampal-neocortical connectivity during memory encoding and postencoding rest in humans. Proceedings of the National Academy of Sciences. 107:7550-7555.

van Kesteren MTR, Rijpkema M, Ruiter DJ, Fernández G. 2010 b. Retrieval of associative information congruent with prior knowledge is related to increased medial prefrontal activity and connectivity. Journal of Neuroscience. 30:15888-15894.

Vrijsen JN, van Amen CT, Koekkoek B, van Oostrom I, Schene AH, Tendolkar I. 2018. Childhood trauma and negative memory bias as shared risk factors for psychopathology and comorbidity in a naturalistic psychiatric patient sample. Brain and Behavior. 8:12.

Knott LM, Thorley C. 2014. Mood-congruent false memories persist over time. Cognition and Emotion. 28:903-912.

Lemogne C, Delaveau P, Freton M, Guionnet S, Fossati P. 2012. Medial prefrontal cortex and the self in major depression. Journal of Affective Disorders. 136:e1-e11.

MacQueen G, Frodl T. 2011. The hippocampus in major depression: evidence for the convergence of the bench and bedside in psychiatric research? Molecular Psychiatry. 16:252-264.

Maquet P, Péters J-M, Aerts J, Delfiore G, Degueldre C, Luxen A, Franck G. 1996. Functional neuroanatomy of human rapideye-movement sleep and dreaming. Nature. 383:163.

Matt GE, Vázquez C, Campbell KW. 1992. Mood-congruent recall of affectively toned stimuli: a meta-analytic review. Clinical Psychology Review. 12:227-255.

Mcclelland JL. 2013. Incorporating rapid neocortical learning of new schema-consistent information into complementary learning systems theory. Journal of Experimental Psychology: General. 142:1190-1210.

McGaugh JL. 2004. The amygdala modulates the consolidation of memories of emotionally arousing experiences. Annual Review of Neuroscience. 27:1-28.

Nishida M, Pearsall J, Buckner RL, Walker MP. 2009. REM sleep, prefrontal theta, and the consolidation of human emotional memory. Cerebral Cortex. 19:1158-1166.

Palagini L, Baglioni C, Ciapparelli A, Gemignani A, Riemann D. 2013. REM sleep dysregulation in depression: state of the art. Sleep Medicine Reviews. 17:377-390.

Ramel W, Goldin PR, Eyler LT, Brown GG, Gotlib IH, McQuaid JR. 2007. Amygdala reactivity and mood-congruent memory in individuals at risk for depressive relapse. Biological Psychiatry. 61:231-239.

Roediger H, Mcdermott K. 1995. Creating false memories: remembering words not presented in lists. Journal of Experimental Psychology: Learning, Memory, and Cognition. 21:803-814.

Ruci L, Tomes JL, Zelenski JM. 2009. Mood-congruent false memories in the DRM paradigm. Cognition and Emotion. 23:1153-1165.

Schmaal L, Veltman DJ, van Erp TGM, Sämann PG, Frodl T, Jahanshad N, Loehrer E, Tiemeier H, Hofman A, Niessen WJ et al. 2016. Subcortical brain alterations in major depressive disorder: findings from the ENIGMA major depressive disorder working group. Molecular Psychiatry. 21:806-812. 
Siegle GJ, Thompson W, Carter CS, Steinhauer SR, Thase ME. 2007. Increased amygdala and decreased dorsolateral prefrontal BOLD responses in unipolar depression: related and independent features. Biological Psychiatry. 61:198-209.

Stickgold R. 2005. Sleep-dependent memory consolidation. Nature. 437:1272-1278.

Thapar A, McDermott KB. 2001. False recall and false recognition induced by presentation of associated words: effects of retention interval and level of processing. Memory \& Cognition. 29:424-432.

Tse D, Langston RF, Kakeyama M, Bethus I, Spooner PA, Wood ER, Witter MP, Morris RGM. 2007. Schemas and memory consolidation. Science. 316:76-82.
Volman I, Roelofs K, Koch S, Verhagen L, Toni I. 2011. Anterior prefrontal cortex inhibition impairs control over social emotional actions. Current Biology. 21:1766-1770.

Warren DE, Jones SH, Duff MC, Tranel D. 2014. False recall is reduced by damage to the ventromedial prefrontal cortex: implications for understanding the neural correlates of schematic memory. Journal of Neuroscience. 34:7677-7682.

Westermann R, Spies K, Stahl G, Hesse FW. 1996. Relative effectiveness and validity of mood induction procedures: a metaanalysis. European Journal of Social Psychology. 26:557-580.

Zhang W, Gross J, Hayne H. 2017. The effect of mood on false memory for emotional DRM word lists. Cognition and Emotion. 31:526-537. 